$30 \mid 2003$

Les Aventures de Télémaque. Trois siècles

d'enseignement du français. I.

\title{
Stratégies de lecture et pédagogie: le cas de la sagesse dans Télémaque
}

\section{Rachele Raus}

\section{(2) OpenEdition Journals}

Édition électronique

URL : https://journals.openedition.org/dhfles/1540

DOI : $10.4000 /$ dhfles. 1540

ISSN : 2221-4038

Éditeur

Société Internationale pour l'Histoire du Français Langue Étrangère ou Seconde

Édition imprimée

Date de publication : 1 juin 2003

Pagination : 72-83

ISSN : 0992-7654

Référence électronique

Rachele Raus, «Stratégies de lecture et pédagogie: le cas de la sagesse dans Télémaque », Documents pour l'histoire du français langue étrangère ou seconde [En ligne], 30 | 2003, mis en ligne le 01 janvier 2012, consulté le 27 mai 2021. URL : http://journals.openedition.org/dhfles/1540 ; DOI : https:// doi.org/10.4000/dhfles.1540

Ce document a été généré automatiquement le 27 mai 2021.

(C) SIHFLES 


\title{
Stratégies de lecture et pédagogie: le cas de la sagesse dans Télémaque
}

\author{
Rachele Raus
}

\section{Préliminaires}

1 Nous voudrions commencer par définir le concept de stratégie de lecture tel que nous l'avons utilisé dans notre intervention. La notion peut se définir comme l'ensemble des mécanismes interprétatifs adoptés par un lecteur lorsqu'il actualise le message d'un texte (Melizzi 1996 : 261). La stratégie de lecture se poserait donc du côté de l'intentio lectoris (Eco 1995), notion qui renvoie à l'interprétation qu'un lecteur donne d'un texte en relation à ses pulsions, à ses connaissances... Cependant, nous voudrions reformuler la notion de stratégie de lecture du côté de l'intentio auctoris (Eco 1995), la reconsidérant non pas dans le sens de l'une des possibles actualisations de lecture d'un texte, mais dans le sens de la stratégie textuelle mise en place par un auteur pour orienter la lecture de son texte et favoriser, par conséquent, une actualisation particulière du texte lui-même. Dans sa stratégie, l'auteur inscrirait le lecteur à l'intérieur du texte, en orientant sa lecture à travers la cohérence narrative et pragmatique. Nous allons donc analyser d'abord (1.) la cohérence narrative dans Les aventures de Télémaque ${ }^{1}$ de Fénelon en utilisant notamment la sémantique structurale de Greimas. Ensuite (2.), en nous servant du traitement automatique du texte et de l'analyse du discours française ${ }^{2}$, nous observerons la cohérence pragmatique. Nous opèrerons une généralisation qui nous permettra de mettre en évidence, par delà la réception du texte à son époque, les possibilités d'un texte qui en fait reste fondateur ${ }^{3}$ en pédagogie, considérant cette dernière en tant qu'étude de l'éducation voire de la formation de l'individu.

\section{Cohérence narrative}

2 À la suite de la sémantique structurale (Greimas 1986, Courtés 1976 et 1989), nous avons vu à l'œuvre, dans Télémaque, une cohérence narrative qui garantit la progression 
thématique et autorise la description d'une action résumable de la façon suivante : le sujet, Télémaque, veut retrouver l'objet du désir, son père Ulysse. Dans cet effort, il est aidé par Minerve et par d'autres adjuvants (Nestor, le prêtre d'Apollon Termosiris,...) et entravé par des opposants (ex. Calypso, Venus, Neptune...). C'est en effet la quête du père qui fait déclencher l'action et qui la résume, puisque le voyage se termine justement avec la rencontre de Télémaque et d'Ulysse. Cela si l'on reste à la surface du texte, mais si nous considérons ce qui se passe du point de vue du parcours sémique, on constate une forte isotopie qui se crée autour de l'axe sagesse-paternité : à bien regarder, en effet, Ulysse n'est autre que l'une des formes de la sagesse. En ce sens, il est à préciser que dans Télémaque il y a plusieurs réalisations de la sagesse : toutes les facettes $^{4}$ du signe " sagesse » se réalisent en Minerve, la déesse de la sagesse, la fille de Jupiter, la déesse qui inspirait déjà l'ars maieutica de Socrate ${ }^{5}$. Outre la sagesse " céleste ", il y a deux autres réalisations de la sagesse : celle du roi / gouvernement; et celle de l'homme en général. La réalisation du roi sage est Ulysse, qui a été « le plus sage de tous les rois de la Grèce ", dit Télémaque (Fénelon 1824 : 31) ; en cela, Ulysse n'est comparable qu'à Minos, "le plus sage et le meilleur de tous les rois ", en dit Mentor (ib. : 33). Minos est d'autant plus sage qu'il a gouverné par de "sages lois » (ib.: 30). Mais il y a, au-delà de la sagesse d'un roi, la sagesse que tout homme peut acquérir, comme celle des Crétois, ou celle par laquelle Ulysse lui-même est non seulement un roi sage, mais, dit Mentor, « le plus sage de tous les hommes » (ib. : 191).

Par cette voie, alors, la véritable quête n'est pas seulement la quête du père, mais plutôt du père en tant que symbole de la sagesse. Cela nous est confirmé également par le fait que Télémaque appelle "père » les sages personnages qui lui servent de guide : Mentor est défini le « vrai père » (ib. : 53), mais, comme le fait remarquer Cuche (1995: 189-192) l'appellatif de père est aussi attribué à Nestor, Termosiris... La véritable macro-action que nous dégageons dans Télémaque devient alors plutôt celle de la transformation d'un sujet qui acquiert la sagesse humaine à son plus haut degré, à savoir la sagesse royale. Rappelons brièvement que pour qu'il y ait action selon Greimas, il faut un sujet qui réalise cette action, passant d'un sujet d'état à un sujet de faire. Pour cela, le sujet doit acquérir une compétence et ne résulte actualisé qu'une fois cette compétence acquise. Nous pourrions schématiser cette transformation de la façon suivante :

$S V \rightarrow S A \rightarrow S R$

où :

$6-S V=$ sujet virtuel : il doit acquérir une compétence pour effectuer une performance ;

$7 \quad-S A=$ sujet actuel qui a acquis une compétence ;

$8-S R=$ sujet réalisé qui effectue la performance.

9 Dans Télémaque ce parcours se présente de la façon suivante :

$10 S V$ (Télémaque) -> SA (Télémaque acquiert la sagesse humaine) / SR (Télémaque peut se réunir à Ulysse / la sagesse royale),

11 où la performance coïncide en fait avec la compétence, Ulysse étant justement le symbole de la sagesse humaine à son plus haut degré (sagesse royale). Au fond, alors il n'y a pas de véritable action, l'action de Télémaque, sa performance, se réduisant à l'acquisition d'une compétence.

Les véritables sujets de faire sont alors les dieux : ce sont eux qui poussent les hommes à agir d'une certaine façon, qui les trompent pour leur bien, qui destinent Télémaque à 
de grandes actions. Ce sont eux les véritables moteurs de l'action : Vénus qui intervient par Neptune ou bien par Cupidon, Minerve qui assure l'instruction de Télémaque...

Le véritable rôle narratif de Télémaque est alors celui de donner au texte une cohérence narrative : c'est de ses aventures que l'on parle, d'où le titre du texte, et c'est lui le véritable destinataire de toute action, du faire des dieux. Même là où il n'est pas présent dans la narration, comme par exemple dans l'épisode de Salente entre Mentor et Idoménée, il est pourtant la raison de cette apparente digression, comme le précise Fénelon :

Minerve [...] établissait ainsi dans Salente toutes les meilleures lois et les plus utiles maximes de gouvernement, moins pour faire fleurir le royaume d'Idoménée que pour montrer à Télémaque, quand il reviendrait, un exemple sensible de ce qu'un sage gouvernement peut faire... (Fénelon $1824: 116$ ).

Ce qui se passe alors, c'est que par la narration des aventures de Télémaque Fénelon crée, au niveau de la cohérence narrative, une tension interne ${ }^{6}$, un plaisir qui invitent à la lecture par une attente (Télémaque réussira-t-il à retrouver Ulysse / la sagesse), stimulant en outre le jeu de rôle du lecteur avec le personnage.

\section{Cohérence pragmatique}

Du point de vue de la cohérence pragmatique, il faut considérer l'oeuvre comme un message adressé à quelqu'un censé être à l'extérieur du texte. Il est clair que le lecteur empirique de Télémaque n'est autre que le duc de Bourgogne, dont Fénelon était précepteur. Pourtant, il nous semble qu'il faut faire la distinction entre le destinataire de la transformation narrative (Télémaque) et le lecteur inscrit dans le texte, destinataire de l'acte pragmatique. En effet, même si la relation à la sagesse se fait essentiellement par la figure de Télémaque et par la relation dialogique entre Télémaque et Mentor, Fénelon interagit, pour ainsi dire, avec le lecteur par Télémaque mais pas seulement lui : ainsi en va-t-il pour le dialogue Mentor-Idoménée, où Télémaque est le destinataire des effets du dialogue, mais non pas du dialogue en soi.

Si Télémaque donne une cohérence narrative au texte, c'est bien le lecteur, lecteur empirique aussi bien que modèle, qui garantit la cohérence pragmatique. Ce qui nous intéresse ici, c'est de voir qui est ce lecteur modèle que le texte envisage par delà le lecteur empirique. Pour ce faire, nous avons privilégié à l'intérieur du texte les occurrences du champ lexical concernant la « sagesse » (« sage » adjectif et substantif ; « sagesse ») et des mots qui co-occurrent avec elles (\$ 2.1); ensuite (\$ 2.2), nous avons analysé la façon dont Fénelon met en place un discours cohérent autour de cette même sagesse.

\subsection{Les occurrences et les co-occurrences}

La notion de « sagesse » se développe dans Télémaque par l'adjectif / substantif « sage » et par le substantif « sagesse ». D'après les occurrences de ces trois mots dans le texte, nous pouvons retracer les cooccurrences suivantes:

18 a) sage $(s)+\operatorname{vieillard}(s)$ : cette cooccurrence relie la sagesse à l'âge adulte, notamment parce que la vieillesse suppose aussi l'expérience, raison pour laquelle l'adjectif apparaît essentiellement lié à des gens experts, comme Ulysse, Mentor, Minos, Nestor et aux rois ; 
) sage (s) + discours / maxime(s) / conseils : c'est en particulier Mentor qui donne des « sages conseils ». En tout cas, cela renvoie au fait que la véritable sagesse n'est pas un bien qu'il faut occulter, mais bien qu'il faut partager avec les autres, le sage devenant une sorte de guide moral ;

sagesse + vertu (+ gloire) : la vertu est l'un des véritables dons qui permettent à l'homme d'être heureux, et qui à son tour co-occurre avec tant d'autres qualités positives (amour pour, courage, justice, confiance, raison, mérite, sincérité, liberté, vérité). L'homme sage se veut aussi l'homme vertueux. Mais si la sagesse adopte des conseils et des maximes, et reste liée à la raison, la vertu s'apprend par des " principes " et des " règles ", suivant un "sentier rude et âpre " (Fénelon 1824 : 151). Jamais Fénelon ne nous donne de définitions explicites de ces notions, mais c'est justement par le cotexte et par le parcours cohérent qu'il crée autour de ces mêmes notions, que nous décelons leur valeur sémantique.

21 Il est aussi important de remarquer que la " sagesse " s'oppose aux " plaisirs » considérés comme des passions déréglées : ainsi, par exemple, la sagesse des sages crétois est « délivré[e] des folles passions et des caprices de la jeunesse » (ib. : 37). Pour acquérir la sagesse, il ne faut pas éliminer les passions, il s'agit plutôt de les maîtriser. L'idéal est donc la modération, déjà exaltée dans l'antiquité : « une vie sobre, modérée, simple, exempte d'inquiétudes et de passions, réglées et laborieuse, retient dans les membres d'un homme sage la vive jeunesse » (ib. : 72). Cette fois, le conseil de Mentor est donné non pas à Télémaque, mais à Idoménée, car c'est dans la relation avec Idoménée que la négativité des passions déréglées se fait plus évidente. Voilà pourquoi alors, « sage » co-occurre aussi avec « modéré " / " modération » et avec " raison ", cette dernière étant conçue comme capacité de l'homme de se conduire de façon modérée.

C'est la sagesse qui donne la véritable joie et qui reste l'idéal à atteindre. Ainsi, si on analyse l'adjectif « heureux », on se rend compte de combien la sagesse est liée à cette autre notion. Tout particulièrement, nous avons observé la formule « heureux X qui / que ", ainsi que son autre formulation " que X sera heureux si », toujours par rapport à la sagesse. Là aussi, nous retrouvons la coprésence de ces deux sagesses, du roi (« Heureux le peuple qui est conduit par un sage roi !", ib. : 9) et de l'homme ("que tu [Télémaque] seras heureux si tu surmontes tes malheurs et si tu ne les oublies jamais ", $i b$. : 12). Télémaque souligne à plusieurs reprises le fait que c'est par la modération des passions, et donc par la sagesse, qu'on peut vraiment être heureux : « heureux ceux qui se dégoûtent des plaisirs violents » (Fénelon $1824: 12$ ), « heureux celui qui se borne à une condition privée et paisible » (ib. : 148)... Au contraire, la passion effrénée rend malheureux, provoquant la mort ou bien les invocations à la mort : ainsi Calypso invoque la mort pour échapper à la passion qu'elle conçoit pour Télémaque; Philoctète blessé invoque la mort en tant que seul remède à la douleur...

Enfin, la cooccurrence avec des mots appartenant aux champs lexicaux de la «bonté » et de la « vérité " place la sagesse parmi les grands idéaux, contribuant à sa connotation positive ; mais aussi à la déterminer comme le véritable bien que l'homme doit chercher. Ainsi, sur l'axe paradigmatique, le roi sage est souvent substitué par le «bon roi ", tout comme l'on retrouve les syntagmes "bon père ", " bonnes lois ", " bon conseil », « bonnes maximes », « bon gouvernement ", à côté ou bien en substitution de l'adjectif « sage ». 
Bref, autour de la « sagesse ", de la " vertu », de la " raison » / " modération » et du couple des antonymes " heureux-malheureux ", Fénelon crée un véritable parcours de lecture cohérent, une stratégie qui fait concevoir la sagesse comme modération, comme maitrise des passions qu'on a connues, et donc comme la seule qui rende l'homme véritablement heureux. L'homme sage, qui a appris à dominer les passions, est celui qui est heureux. Le roi sage est heureux en tant qu'homme et fait le bonheur de son peuple, mais il est aussi malheureux parce qu'il est confronté aux passions, la flatterie entre autres, des hommes qu'il conduit.

\subsection{Le discours autour de la sagesse : l'axe Mentor-Télémaque}

Le discours autour de la sagesse, considéré non seulement comme objet explicite à l'intérieur du discours, mais comme objet qui, par une série d'indices dans le texte, et notamment l'adjectif " sage ", se distribue tout au long du texte pour lui donner une cohérence pragmatique, s'articule essentiellement autour de quatre axes :

- le dialogue entre Mentor et Télémaque ;

- le dialogue entre Télémaque et les Crétois / Télémaque et les princes alliés ;

- le dialogue entre Idoménée et Mentor ;

- la présence de l'auteur omniscient.

Nous examinons ici le premier de ces quatre axes.

Le dialogue entre Télémaque et Mentor se caractérise par l'énoncé de Fénelon, qui, auteur omniscient, commente la situation de Télémaque après qu'il a quitté Calypso : « il [Télémaque] sentait le besoin de suivre toujours les sages conseils de Mentor " (Fénelon 1824 : 57). Le dialogue entre Mentor et Télémaque se fonde alors sur un objet de discours qui, explicité ou non, reste pourtant l'objet qui justifie la démarche dialogique : la sagesse, que Mentor possède et que Télémaque doit acquérir. Ce dialogue présente en général des caractéristiques assez précises : d'un côté, le discours de Mentor se veut assertif, de l'autre, celui de Télémaque reste très modalisé dans le sens du doute.

Mentor assère notamment par le recours à trois procédés assertifs :

1) les maximes : il s'agit de maximes qui découlent du sens commun et des faits observés (à propos des maximes, nous renvoyons le lecteur à Papasogli 2000) ;

2) la modalité déonthique de nécessité :

a) par le recours à l'expression "il faut ", soit dans les maximes générales soit dans celles qui sont adressées directement à Télémaque. En voici quelques exemples : « il faut se soutenir par le courage » (ib.: 5) ; « avant que de se jeter dans le péril, il faut le prévoir et le craindre » (ib.) ; "il faut que tu [Télémaque] deviennes grand par la patience » $(i b$.$) ; « il faut que ce soit la sagesse et la vertu, plutôt que la présence de$ Mentor, qui vous [Télémaque] inspire ce que vous devez faire » (ib. : 91)...

b) par le recours à l'impératif comme dans les cas suivants : "Songez plutôt à soutenir la réputation de votre père " (ib. : 3$)$; dans la tirade lors de l'épisode de Calypso : « Craignez [...] craignez [...] Gardez-vous bien de [...] Gardez-vous [...] craignez [...] attendez toujours $[\ldots] »(i b .: 3) \ldots$

3) les questions rhétoriques, reposant sur des présupposés moraux qui orientent la lecture, et qui donnent des enseignements de vie. Par exemple, dans les énoncés 
suivants : « Est-ce donc là, ô Télémaque, les pensées qui doivent occuper le cœur du fils d'Ulysse? » (ib.) ; « que feriez-vous d'une vie immortelle, sans liberté, sans vertu et sans gloire? » (ib. : 49)...

Le dit de Télémaque par contre est dubitatif, reposant sur le questionnement et l'incertitude, notamment par :

1) le recours à une modalité non assertive :

a) par le recours au connecteur " peut-être », comme dans ces quelques énoncés : «Je vais parler à Hasaël ; il aura peut-être pitié de ma jeunesse et de mes larmes » (ib. : 31); "Vous me blâmerez peut-être de prendre trop facilement des inclinations dans les lieux où je passe » (ib.: 177)...

b) par l'utilisation du verbe " pouvoir » comme, par exemple, dans les énoncés "Quoi donc ! pouvais-je refuser à Calypso de lui raconter mes malheurs ?» (ib. : 26) ; « que peuvent donc signifier ces dernières paroles [...] ?» (ib.: 73)

42 2) le recours à de vraies questions : « Mais que ferai-je donc ? ( $i b .: 26)$; « mais que peuvent donc signifier ces dernières paroles : Tu reverras ...?» (ib. : 73)... Souvent ces questions soulignent, par l'implicite, la relation de guide du maître : " que ferais-je maintenant sans vous ?» (ib. : 30) ; « Mon cher Mentor, pourquoi ai-je refusé de suivre vos conseils? » (ib. : 5)...

43 3) l'émotion, tout particulièrement sous forme de soupirs et d'invocations, comme dans : « Hélas ! [...] il n’y a plus d'Ithaque pour nous; nous ne reverrons jamais ni notre patrie, ni Pénélope [...] Mourons, mon cher Mentor » (ib. : 9); « Hélas ! d'où venez-vous ! En quels dangers ne m'avez-vous point laissé pendant votre absence! » (ib. : 30)...

Dans deux cas seulement, le discours de Télémaque se pose sur un plan général et se fait assertif :

1) avec les Crétois, mais il précise qu'il est inspiré par Mentor ;

2) avec les Princes alliés, mais dans l'épisode la transformation de Télémaque en sujet actualisé est en cours.

Le questionnement de Télémaque à Mentor est une ressource pour co-construire l'objet de discours (Mondada 1994 et 2000), qui reste, somme toute, une morale à apprendre, fondée sur la sagesse. Pour cela le discours entre Mentor et Télémaque est « polémique " (Orlandi 1984: 6) dans la mesure où justement le questionnement de Télémaque permet à Mentor d'avoir un appui sur lequel porter son argumentation, et que donc c'est à partir du dit de Télémaque que Mentor intervient pour orienter le discours ; par conséquent l'objet de discours résulte comme co-construit par les deux interlocuteurs. Ainsi en va-t-il, par exemple, dans le dialogue suivant :

[Mentor] Un prince sage, qui ne veut que le bon ordre et la justice, parviendra, avec le temps, à se passer des hommes corrompus et trompeurs; il en trouvera assez de bons qui auront une habileté suffisante. Mais ce n'est pas assez de trouver de bons sujets dans une nation, il est nécessaire d'en former de nouveaux.

- Ce doit être - répondit Télémaque - un grand embarras.

- Point du tout - reprit Mentor - l'application que vous avez à chercher les hommes habiles et vertueux, pour les élever, excite et anime tous ceux qui ont du talent et du courage ; chacun fait des efforts (Fénelon 1824 : 187).

Tout de même, le questionnement repose sur un discours « autoritaire » (Orlandi 1984 : 7), où les grands principes moraux illustrés par Mentor ne sont jamais mis en doute et fondent le discours en tant que présupposés partagés. Ainsi en va-t-il pour le dialogue 
que nous venons de citer, où la question de Télémaque ne porte pas sur la nécessité du roi sage de former de bons sujets afin de garder le bon ordre, mais sur la difficulté de former ces individus, les maximes implicites selon lesquelles « le roi sage veut le bon ordre et la justice " et que pour cela " il faut que le roi sage forme de bons sujets " fondant le dialogue sur des présupposés partagés. En voici encore un autre exemple :

[Mentor] Quand est-ce, ô Télémaque, que vous serez assez sage pour ne parler jamais par vanité et que vous saurez taire tout ce qui vous est avantageux, quand il n'est pas utile à dire ? [....]

- Quoi donc ! - répondit Télémaque - pouvais-je refuser à Calypso de lui raconter mes malheurs?

- Non - reprit Mentor - il fallait les lui raconter : mais vous deviez le faire en ne lui disant que ce qui pouvait lui donner de la compassion. Vous pouviez dire que vous aviez été tantôt errant, tantôt captif en Sicile, et puis en Égypte. C'était lui dire assez, et tout le reste n'a servi qu'à augmenter le poison qui brûle déjà son cœur. Plaise aux dieux que le vôtre puisse s'en préserver!

- Mais que ferai-je donc ? - continua Télémaque d'un ton modéré et docile. (ib. : 26).

Dans l'échange, il n'est jamais question de mettre en cause le principe selon le quel « il ne faut jamais parler par vanité ». Télémaque au début ne semble pas le comprendre, mais ensuite il lui semblera évident au point de le pousser à suivre le «bon conseil » de son ami. Les maximes de Mentor sont sages par antonomase et il suffit qu'elles soient illustrées par l'exemple pour les faire accepter :

[Télémaque] J'éprouve - s'écriait-il parlant à Mentor - ce que vous me disiez et que je ne pouvais croire, faute d'expérience : on ne surmonte le vice qu'en le fuyant (ib. : 56).

Pourtant, il peut arriver que l'évidence ne suffise pas, le parcours pour acquérir la sagesse étant parfois difficile. Ainsi, peu avant la fin du livre :

Télémaque, agité au-dedans par une peine secrète, semblait n'avoir jamais compris ces maximes, quoiqu'il en fût rempli et qu'il les eût lui-même enseignées aux autres. Une humeur noire lui donnait, contre ses véritables sentiments, un esprit de contradiction et de subtilité pour rejeter les vérités que Mentor expliquait. (ib. : 189).

51 Mais le narrateur omniscient fait de cet épisode l'exception confirmant la règle : les enseignements de Mentor sont des « vérités » et si Télémaque ne le voit pas, c'est parce qu'il est pris par un esprit de contradiction qui entrave ses véritables sentiments.

Outre les présupposés moraux, il y a encore une autre forme d'implicite qu'il nous faut relever, à savoir un sous-entendu ${ }^{7}$ qui rend possible tout discours sur la sagesse : le fait que Télémaque veuille acquérir cette sagesse. Voilà pourquoi, lorsque Télémaque invoque la mort, ou bien renonce à la quête, Mentor intervient de façon autoritaire et accuse Télémaque, d'être le fils indigne d'Ulysse. A ce moment là, la progression du dialogue s'arrête, Mentor agissant par un acte d'autorité, puisque ce qui manque est la condition même de toute instruction. Ainsi en va-t-il, par exemple, lorsque Télémaque propose à Mentor de ne pas quitter l'île de Calypso, ce dernier l'accusant alors en ces termes : " Lâche fils d'un père si sage et si généreux, menez ici une vie molle et sans honneur au milieu des femmes!» (ib. : 49) ; de même, lorsque Télémaque invoque la mort lors de la captivité chez les Égyptiens, Mentor s'écrie : "Indigne fils du sage Ulysse ! Quoi donc! vous vous laissez vaincre à votre malheur !» (ib. : 9)...

53 À partir de ce que l'on vient de dire, nous pouvons voir qu'à travers les cooccurrences autour de "sage-sagesse » et les implicites dégagés sur l'axe Mentor- Télémaque, Fénelon oriente fortement l'actualisation d'une certaine lecture de son texte et nous 
fournit des indices importants pour ce qui est de son lecteur modèle. Ce qui se dégage du point de vue de la lecture orientée, c'est que Fénelon légitime la nécessité de l'acquisition de maximes "sages ", et qu'il suppose par là un élève jeune et docile qui, comme Télémaque, veuille les acquérir. C'est bien cet élève qui s'avère être le lecteur modèle de l'oeuvre de Fénelon : Télémaque se révèle être alors un outil précieux de scolarisation, c'est-à-dire de l'« intégration de l'enfant dans le monde scolaire " (Verdelhan-Bourgade 2002 : 51), contribuant, par les valeurs qu'il met en place, à constituer l'élève auquel il s'adresse. L'utilisation de la fiction littéraire, stimulant en ce sens la structuration de l'identité par l'émotion et le jeu de rôle (cf. ELA 1999), favorise l'intériorisation des relations constitutives de l'acte pédagogique, à savoir les relations élève-maître et élève-savoir, et par là l'intériorisation de la scolarisation. Ainsi, donc, la cohérence narrative produirait une tension interne invitant à la lecture (Télémaque réussira-t-il à retrouver Ulysse / la sagesse ?), lecture qui, par la cohérence pragmatique, est ensuite orientée de façon à créer une tension externe ${ }^{8}$, faisant ressentir comme indispensable l'acquisition de la sagesse, qui seule fait le véritable bonheur de l'homme.

Il est indiscutable qu'au delà de la sagesse humaine, le parcours esquissé dans Télémaque vers le degré le plus parfait de la sagesse humaine, la sagesse royale, visait le Duc de Bourgogne, comme lecteur empirique. Tout de même, dans la mesure où Télémaque non seulement " réfléchit explicitement sur l'acte pédagogique » (Cuche 1995 : 40) mais qu'il le fonde comme légitime en stimulant l'intériorisation du modèle scolaire, le texte reste très actuel à n'importe quelle époque pour tout élève et se révèle fondateur en pédagogie.

\section{Conclusion}

Si, alors, Télémaque a ce rôle essentiel dans la culture occidentale, c'est bien par une stratégie de lecture qui vise la scolarisation de l'élève, stratégie qui traverse les époques, posant l'acquisition de la sagesse comme but fondamental pour n'importe quel lecteur. En ce sens, nous voudrions terminer sur une réflexion que la sagesse nous pose en tant que véritable signe polysémique s'actualisant différemment selon les époques.

Nous avons eu l'occasion (Raus 2001) de retracer dans des textes de voyage du XVI siècle et jusqu'à la première partie du XVII ${ }^{e}$ siècle, un syllogisme, concernant Ulysse, qui peut se résumer de la façon suivante :

1- la vue fait acquérir la connaissance (Aristote) ;

2- Ulysse a vu plusieurs villes et mœurs (Homère) ;

3- Ulysse a acquis la connaissance (ou bien : Ulysse est devenu sage).

Selon le syllogisme, la sagesse découlerait, donc, de la connaissance de l'altérité : un savoir faire (le voyage et la rencontre de l'autre) permettrait d'acquérir la connaissance, autrement dit un savoir faire permettrait un faire savoir. A la fin du XVII ${ }^{\mathrm{e}}$ siècle, Fénelon renverse ce paradigme et, faisant découler la connaissance des autres de la connaissance de soi-même, met en place un faire savoir (l'acquisition de la sagesse) comme nécessaire pour l'acquisition de tout savoir faire (bien gouverner). Il nous semble qu'il y a là un fort changement d'episteme : l'itinéraire pédagogique, comme voyage à l'intérieur de soi, se substituerait bien à l'itinéraire de voyage en tant que découverte de l'autre. 


\section{BIBLIOGRAPHIE}

\section{Bibliographie}

BOUCHARD, R. (1985), «Interaction, discours et tension », in D. VÉRONIQUE et R. VION dir., Modèles de l'interaction verbale, Publications de l'Univ. de Provence.

CICUREL, Francine (2000), « Dispositifs textuels et persuasion clandestine », ELA 119, 291-304.

COSSUTTA, Frédéric (2000), « Lire / Élire les philosophes », ELA 119, 373-384.

COURTÉS, Joseph (1976), Introduction à la sémiotique narrative et discursive, Paris, Hachette.

COURTÉS, Joseph (1989), Sémantique de l'énoncé : applications pratiques, Paris, Hachette.

CUCHE, François-Xavier (1995), Télémaque entre père et mer, Paris, Champion.

DUCROT, Oswald (1993), Dire et ne pas dire, Paris, Hermann.

ECO, Umberto (1979), Lector in fabula, Milano, Bompiani.

-- (1995), Interpretazione e sovrainterpretazione, Milano, Bompiani.

ELA (1999), Fiction littéraire et apprentissage des langues, 115.

FÉNELON (1824), Les aventures de Télémaque, édition établie par Jeanne-Lydie Goré, www.bnf.fr (section Gallica).

GREIMAS, Algédiras Julien (1986), Sémantique structurale, Paris, PUF.

GUILHAUMOU, Jacques, MALDIDIER, Denise, ROBIN, Régine (1994), Discours et archive, Liège, Mardaga.

KLEIBER, Georges (1999), Problèmes de sémantique : la polysémie en question, Villeneuve, Presses Universitaires du Septentrion.

LAMAISON, Jacques (s.d.), Les aventures de Télémaque, avec une notice biographique, une notice historique et littéraire, des notes explicatives, des jugements, un questionnaire sur la pièce et des sujets de devoirs, Paris, Classiques Larousse.

MAINGUENAU, Dominique (1984), Genèses du discours, Liège, Mardaga.

-- (1993), Le Contexte de l'œuvre littéraire, Paris, Dunod.

-- (1997), L’Analyse du discours, Paris, Hachette.

-- (2000), « Lecture, incorporation et monde éthique », ELA 119, 265-275.

MAINGUENAU, Dominique, COSSUTTA, Frédéric (1995), «L'analyse des discours constituants », Langages 117, 112-125.

MAUGAIN, Gabriel (1910), Documenti bibliografici e critici per la storia della fortuna del Fénelon in Italia, Paris, Champion.

MELIZZI, Roberto (1996), Nuovi apporti della linguistica - Aspetti teorici e pratici, Pescara, Libreria dell'Università di Pescara Editrice.

MONDADA, Lorenza (1994), Verbalisation de l'espace et fabrication du savoir-Approche linguistique de la construction des objets de discours, Lausanne, Université de Lausanne. 
-- (2000), Décrire la ville. La construction des savoirs urbains dans l'interaction et dans le texte, Paris, Anthropos.

ORLANDI, Eni (1984), « Typologie du discours et règles de la conversation », Langage et Société 29, 3-25.

PAPASOGLI, Benedetta (2000), « Le massime della sapienza nel Télémaque di Fénelon », in N. MINERVA e C. PELLANDRA dir., Aspetti di etica applicata. La scrittura aforistica, Bologna, CLUEB, $15-28$.

RAUS, Rachele (2001), « Pour une approche interdisciplinaire du stéréotype : le cas du topos d'Ulysse au XVIe siècle », in Le stéréotype : formes, usages et stratégies, Actes du XXI colloque d'Albi « Langage et Signification », Toulouse, CALS, 95-106.

VERDELHAN-BOURGADE, Michèle (2002), « Le manuel comme discours de scolarisation », ELA 125, 37-52.

\section{NOTES}

1. Nous avons consulté le texte sous un format électronique qui nous a permis de le soumettre à un traitement automatique. L'édition choisie est établie par Jeanne-Lydie Goré sur l'exemplaire de Versailles, en 18 livres, et est déchargeable à l'adresse www.bnf.fr (dans la section Gallica). C'est justement l'exemplaire de Versailles qui a été le plus souvent réédité par les éditeurs modernes, comme précise Jacques Lamaison (s.d. : 6) dans l'édition des classiques Larousse. Pour ce qui est de la numération des pages, nous avons utilisé celle du fichier en format pdf.

2. Pour l'analyse du discours en France, voir en particulier les textes de Mainguenau cités en bibliographie.

3. Pour la notion de texte-discours fondateur, cf. la notion de discours constituant de Mainguenau et Cossutta (1995).

4. On doit à Cruise la notion de facette, qu'il définit comme « une sorte de variation sémantique qui se situe entre la polysémie et la variation contextuelle » (dans la citation de Kleiber 1999 : 90). Kleiber y retrouve une « nouvelle modalité de sens multiple [...] dont l'originalité est de tenir à la fois de la polysémie, par l'autonomie des facettes, et du sens unique, par le caractère unitaire du tout conceptuel » $(1999: 93)$.

5. Le chant de Mentor autour de l'épisode de Minerve sortant de la tête de Jupiter, « c'est-à-dire la sagesse, que ce dieu forme au dedans de lui-même et qui sort de lui pour instruire les hommes dociles » (Fénelon $1824: 62$ ), nous confirme cette filiation par rapport à l'antiquité grecque.

6. Les notions de tension interne-externe se doit à Bouchard (1985) : la tension interne est la tension que le texte suscite par le plaisir de savoir ce qui va se passer par la suite; la tension externe est la tension suscité par une nécessité extérieure au texte qui nous invite à la lecture. Voir aussi Cicurel 2000.

7. Oswald Ducrot définit le sous-entendu comme une forme d'implicite qui « n'est plus à chercher au niveau de l'énoncé, comme un prolongement ou un complément du niveau explicite, mais à un niveau plus profond, comme une condition d'existence de l'acte d'énonciation » (1993 : 9). En ce sens, le sous-entendu se différencie du présupposé, ce dernier étant un implicite « qui fait partie de la signification littérale des énoncés » (ibid.).

8. Voir note 6.

9. D'ailleurs dans le célèbre sonnet de Du Bellay, la formule «Heureux X qui ", que nous avons analysé, se présente de la façon suivante : « heureux qui comme Ulysse, a fait un beau voyage » (Regrets, 1558 : XXXI). 


\section{RÉSUMÉS}

Considérant la notion de stratégie de lecture comme l'actualisation du texte visée par l'auteur, nous avons dégagé le lecteur modèle du Télémaque, pour déterminer les possibles raisons du succès de ce texte en tant qu'outil pédagogique. Prenant la " sagesse " comme observatoire d'analyse, nous avons vu comment, par la cohérence narrative, Fénelon met en place une tension interne au texte qui invite à la lecture. À travers les cooccurrences et les implicites, l'auteur tisse une cohérence pragmatique produisant une tension externe au texte : il rend indispensable l'acquisition de la sagesse et par là il fait de son texte un outil de scolarisation qui, non seulement s'adresse à tout jeune élève docile, mais contribue également à le constituer.

By a redefinition of the reading oriented approach as the author's strategy to actualize one particular reading of his book, we recognized the model reader in Fenelon's Telemachus and defined the reasons for this book's success in pedagogy. We considered « wisdom » as the focus of analysis. We demonstrated that Fenelon uses the narrative coherence to set up an internal tension as a stimulus to reading. By the co-occurrences and the forms of implicits, the author uses the pragmatic coherence to create an external tension : he posits the wisdom acquisition as a basic and necessary skill. So Fenelon turns his book into an essential tool to educate children : not only does he address docile students but also contributes to form them through his book

\section{INDEX}

Keywords : implicits, pedagogy, pragmatic coherence, Reader, reading oriented approach, wisdom

Mots-clés : cohérence pragmatique, implicites, Lecteur, pédagogie, sagesse, stratégie de lecture

\section{AUTEUR}

\section{RACHELE RAUS}

Università di Torino 